Pacific Journal of Mathematics

OPERATORS COMMUTING WITH TRANSLATIONS 


\title{
OPERATORS COMMUTING WITH TRANSLATIONS
}

\author{
R. E. EDWARDS
}

\begin{abstract}
This paper is concerned with the representation, in terms of convolutions with pseudomeasures, of continuous linear operators which commute with translations and which transform continuous functions with compact supports on a Hausdorff locally compact Abelian group $G$ into restricted types of Radon measures on $G$. The two main theorems each assert that any such operator $T$ is of the form $T f=s * f$ for a suitably chosen pseudomeasure $s$ on $G$; the assertions differ in detail in respect of the hypotheses imposed on the range of $T$. The second theorem is an extension of Proposition 2 of [1] from the case in which $G$ is a finite product of lines and/or circles to the general situation.
\end{abstract}

Preliminaries. The notations are as described in $\S 1$ of [1], with $G$ in place of $X$, and with the following additions. If $K \subset G$, $C_{K}(G)$ denotes the set of $f \in C_{c}(G)$ satisfying supp $f \subset K$. The symbol $M_{b}(G)$ will denote the set of all bounded Radon measures on $G$. Continuity of the operators $T$ considered will, in the absence of any indication to the contrary, refer to the inductive limit topology on $C_{c}(G)$ and the vague topology $\sigma\left(M(G), C_{c}(G)\right)$ on $M(G)$ and its subsets. No distinction is drawn between a locally integrable function $f$ on $G$ and the associated measure $f d x \in M(G), d x$ denoting the element of Haar measure on $G$. In this paper, $X$ will denote the character group of $G$, the Haar measure $d \xi$ on $X$ being chosen so that the Fourier transformation is an isometry of $L^{2}(G)$ onto $L^{2}(X)$.

Prior to stating the representation theorems, we make some remarks about pseudomeasures on $G$.

Let $A(G)$ denote the space of functions $u$ on $G$ which are inverse Fourier transforms of functions $v \in L^{1}(X)$ :

$$
u(x)=\int_{X} v(\xi) \xi(x) d \xi \text {; }
$$

$A(G)$ is a Banach space under the norm

$$
\|u\|_{A}=\int_{X}|v(\xi)| d \xi \equiv\|v\|_{1}
$$

By a pseudomeasure on $G$ is meant a continuous linear functional on $A(G)$, and we denote by $P(G)$ the set of pseudomeasures on $G$. By $\|\cdot\|_{P}$ is meant the usual norm on $P(G)$ qua dual of $A(G)$. The Fourier transformation can be defined for pseudomeasures $s$ in such a way that

Received October 15, 1964. 
$s \rightarrow \hat{s}$ is an isometric isomorphism of $P(G)$ onto $L^{\infty}(X)$. There is an obvious sense in which $M_{b}(G)$ can be regarded as a subset of $P(G)$.

If $G$ is a finite product of lines and/or circles, one may think of $P(G)$ as comprising exactly those temperate distributions on $G$ whose Fourier-Schwartz transform is an essentially bounded function. It is this identification which provides the link between Proposition 2 of [1] and Theorem 2 below.

If $s \in P(G)$, the mapping $f \rightarrow s * f$ is a continuous endomorphism of $L^{2}(G)$. In connection with Theorem 1 we shall be concerned with the case in which the restriction of this mapping to $C_{c}(G)$ has a range lying in $M_{b}(G)$, i.e., equivalently, in $L^{1}(G)$. The pseudomeasures $s$ having this property form a subset $P^{1}(G)$ of $P(G)$. Naturally, $P^{1}(G)$ contains the set $P_{c}(G)$ of all pseudomeasures with compact supports (in particular, $P^{1}(G)=P(G)$ when $G$ is compact) and contains also $M_{b}(G)$. The closed graph theorem shows that, if $s \in P^{1}(G)$, then to each compact set $K \subset G$ corresponds a number $m_{k}>0$ such that

$$
\|s * f\|_{1} \leqq m_{K}\|f\| \quad\left(f \in C_{K}(G)\right),
$$

where $\|\cdot\|$ denotes the supremum norm. Further comments on $P^{1}(G)$ are given in $\S 5$ infra.

We can now state the two main theorems.

THEOREM 1. The continuous linear operators $T$ from $C_{c}(G)$ into $M_{b}(G)$ which commute with translations are precisely those of the form

$$
T f=s * f,
$$

where $s \in P^{1}(G)$.

THEOREM 2. The continuous linear operators $T$ from $C_{c}(G)$ into $M_{c}(G)$ which commute with translations are precisely those of the form (1.2), where now $s \in P_{c}(G)$.

Theorem 2, combined with the basic properties of pseudomeasures, shows that any continuous linear operator $T$ from $C_{c}(G)$ into $M_{c}(G)$ which commutes with translations admits an extension which maps $L_{c}^{2}(G)$ into $L_{c}^{2}(G)$ and $L^{2}(G)$ into $L^{2}(G), L_{c}^{2}(G)$ denoting $L^{2}(G) \cap M_{c}(G)$, i.e., the set of functions in $L^{2}(G)$ which vanish a.e. outside a compact subset of $G$ (a property equivalent to saying that the associated measure has a compact support). In $\S 5(\mathrm{~B})$ we shall see that, by virtue of Theorem 1 , each continuous linear operator from $C_{c}(G)$ into $M_{b}(G)$ admits somewhat similar but less evident extensions. 
In Theorem (3.2) of [3] G. I. Gaudry has shown that there is a valid analogue of Theorem 1 for the case in which $M_{b}(G)$ is replaced by the space $M(G)$ of all Radon measures on $G$, the pseudomeasure $s$ being then replaced by a somewhat more general entity termed a "quasimeasure". Theorem 2 above is used in [3] as an aid in studying the local structure of quasimeasures.

2. In the proof of Theorem 1 we shall need a lemma.

Lemma. To each subset $W$ of $G$ containing interior points corresponds a number $c=c_{W}>0$ such that

$$
\|F\|<c \cdot \operatorname{Sup}\left\{\|F \hat{f}\|: f \in C_{W}(G),\|f\| \leqq 1\right\}
$$

for all functions $F$ on $X$.

Proof. Define

$$
N(F)=\operatorname{Sup}\left\{\|F \hat{f}\|: f \in C_{W}(G),\|f\| \leqq 1\right\},
$$

which is possibly $\infty$. If $F$ is unbounded on $X$, the lemma on p. 281 of [1] shows that $N(F)=\infty$, so that in this case any value of $c>0$ will suffice (provided the usual conventions are adopted). Assume then that $F \in B(X)$, the space of bounded functions on $X$. The functional $N$ is evidently a norm on $B(X)$. Moreover, $B(X)$ is complete for $N$. For suppose that $\left(F_{n}\right)$ is an $N$-Cauchy sequence in $B(X)$. Evidently, to each $\xi \in X$ corresponds a number $b_{\xi}>0$ such that

$$
|F(\xi)| \leqq b_{\xi} \cdot N(F) \text {. }
$$

It follows that $\left(F_{n}(\xi)\right)$ is Cauchy for each $\xi \in X$, so that $F=\lim F_{n}$ exists pointwise on $X$. For any $\varepsilon>0$ there exists $n_{0}=n_{0}(\varepsilon)$ such that

$$
N\left(F_{m}-F_{n}\right) \leqq \varepsilon \quad\left(m, n>n_{0}\right) .
$$

That is, for any $f \in C_{W}(G)$ satisfying $\|f\| \leqq 1$,

$$
\operatorname{Sup}_{\xi \in X}\left|F_{m}(\xi)-F_{n}(\xi)\right||\hat{f}(\xi)| \leqq \varepsilon \quad\left(m, n>n_{0}\right) .
$$

On letting $m \rightarrow \infty$ it appears that

$$
\operatorname{Sup}_{\xi \in X}\left|F(\xi)-F_{n}(\xi) \| \hat{f}(\xi)\right| \leqq \varepsilon \quad\left(n>n_{0}\right)
$$

and hence that

$$
N\left(F-F_{n}\right) \leqq \varepsilon \quad\left(n>n_{0}\right) .
$$

This shows first that $N(F)<\infty$, and hence that $F \in B(X)$, and then that $F_{m} \rightarrow F$ in the sense of the norm $N$. Thus $B(X)$ is $N$-complete. 
Reference to (2.1) shows that the supremum norm is lower semicontinuous relative to $N$. Therefore, this supremum norm is actually continuous relative to $N$, which is precisely what the lemma asserts.

3. Proof of Theorem 1. The inequality (1.1) makes it plain that, if $s \in P^{1}(G)$, then (1.2) defines $T$ as a continuous linear operator from $C_{c}(G)$ into $L^{1}(G) \subset M_{b}(G)$ which commutes with translations. Actually $T$, thus defined, maps $C_{c}(G)$ into $L^{2}(G)$ and is continuous for the $L^{2}-$ topologies.

Turning to the converse, let us first show that the seminorm $f \rightarrow \int_{G} d(|T f|)$ is continuous on $C_{c}(G)$. Indeed, integration theory shows that

$$
\int_{G} d(|T f|)=\operatorname{Sup}\left|\int_{G} f d(T f)\right|
$$

the supremum being taken with respect to those $f \in C_{c}(G)$ satisfying $\|f\| \leqq 1$. It thus appears that the seminorm $f \rightarrow \int_{G} d(|T f|)$ is lower semicontinuous on the barrelled space $C_{c}(G)$, and is therefore continuous.

Accordingly, if $K \subset G$ is compact, there exists a number $m_{K}>0$ such that

$$
\int_{\theta} d(|T f|) \leqq m_{K}\|f\| \quad\left(f \in C_{K}(G)\right) .
$$

Take now a net $\left(e_{i}\right)$ of nonnegative functions in $C_{c}(G)$ such that $\int_{G} e_{i} d x=1$ and supp $e_{i} \subset N_{i}$, where the $N_{i}$ form a neighbourhood base at the origin in $G$. We may assume that all the $N_{i}$ are contained in some compact set $N$. If $f \in C_{K}(G)$, then $\lim e_{i} * f=f$ uniformly on $G$ and supp $\left(e_{i} * f\right) \subset N+K$. Since $T$ is continuous and commutes with translations, $T(e * f)=T e * f$ for $e, f \in C_{c}(G)$. So, if $\mu_{i}=T e_{i}$, it follows from (3.1) that

$$
T f=\lim T\left(e_{i} * f\right)=\lim \mu_{i} * f \text { in } M_{b}(G),
$$

and that

$$
\int_{G} d\left(\left|\mu_{i} * f\right|\right) \leqq m_{N+K}\|f\|
$$

Taking the Fourier transform of this relation, it follows that for $f \in C_{K}(G)$ we have

$$
\left\|\hat{\mu}_{i} \cdot \hat{f}\right\| \leqq m_{N+E}\|f\| \text {. }
$$

Fixing $K$ as any compact set with interior points, and applying the lemma in $\S 2$, we conclude that 


$$
\operatorname{Sup}_{i}\left\|\hat{\mu}_{i}\right\|<\infty \text {. }
$$

This in turn ensures that the net $\left(\mu_{i}\right)$ has a weak limiting point $s \in P(G)$. The net $\left(\mu_{i} * f\right)$ then has $s * f$ as a weak limiting point in $L^{2}(G)$ and a comparison with (3.2) shows that $T f$ must coincide with $s * f$, i.e., that (1.2) must hold. Since $T$ maps $C_{c}(G)$ into $M_{b}(G)$, $s$ must belong to $P^{1}(G)$. The proof is complete.

4. Proof of Theorem 2. Once again it is evident that, if $s \in P_{c}(G)$, then (1.2) defines $T$ as a continuous linear map of $C_{c}(G)$ into $M_{c}(G)$ which commutes with translations.

For the converse, note that Theorem 1 implies the existence of a pseudomeasure $s$ such that (1.2) holds. The proof of Theorem 1 shows moreover that $s$ is a weak limiting point in $P(G)$ of the measures $\mu_{i}=T e_{i}$. Now supp $e_{i} \subset N$, a compact subset of $G$. Lemmas 2 and 3 of [2] show that accordingly there is a compact subset $K^{\prime}$ of $G$ such that supp $\mu_{i} \subset K^{\prime}$ for all $i$. But then it follows that supp $s \subset K^{\prime}$ too, showing that $s \in P_{c}(G)$.

REMARK. In Theorem 4.2 of [3] it is remarked that Theorem 2 entails that every quasimeasure with a compact support is a pseudomeasure. Theorem 1 leads to an analogous result, as we now show.

Reference to the proof of Theorem 4.5 of [3] confirms that if $q$ is a quasimeasure on $G$, then $f \rightarrow q * f$ maps $L_{c}^{2}(G)$ continuously into $L_{l o c}^{2}(G)$. Let us write

$$
\|h\|_{1}=\int_{G}^{*}|h(x)| d x \quad(\leqq \infty)
$$

for an arbitrary complex-valued function $h$ on $G$, so that $h \in L^{1}(G)$ if and only if $h$ is measurable and $\|h\|_{1}<\infty$. Then we have the

CoROLlary. If $q$ is a quasimeasure on $G$ such that

$$
\|q * f\|_{1}<\infty \quad\left(f \in C_{c}(G)\right),
$$

then $q$ is a pseudomeasure belonging to $P^{1}(G)$.

Proof. Since $q * f \in L_{l o c}^{2}(G)$, (4.1) shows that $q * f \in L^{1}(G)$. The preceding remarks show that the mapping $f \rightarrow q * f$ has a graph which is closed in $C_{c}(G) \times L^{1}(G)$ and is therefore continuous. The assertion therefore follows from Theorem 1.

5. Concerning $P^{1}(G)$. We collect a few results about $P^{1}(G)$ and its elements.

(A) When $G$ is compact, $P^{1}(G)=P(G)$ (see $\S 1$ ). The situation is 
much more complex when $G$ is noncompact, and we know of no effective and direct characterisation of $P^{1}(G)$ as a subset of $P(G)$. It is easy to see that if $s \in P^{1}(G)$, then $\hat{s}$ coincides l.a.e. on each compact subset $H$ of $X$ with the transform of an ( $H$-dependent) function in $L^{1}(G)$; in particular, $\hat{s}$ is equal l.a.e. on $X$ to a continuous function on $X$. This shows that $P^{1}(G)$ is dense in $P(G)$ if and only if $G$ is compact. More elaborate arguments (based on properties of Helson subsets of $X$; see [4], Chapter 5) will show also that $P^{1}(G)$ is closed in $P(G)$ if and only if $G$ is compact.

We turn next to a positive assertion which adds interest and weight to Theorem 1.

(B) Suppose that $s \in P^{1}(G)$, that $2 \leqq p \leqq \infty$, and that $p^{\prime}$ is defined by $1 / p+1 / p^{\prime}=1$. Let $W$ be any relatively compact open subset of $G,\left(a_{r}\right)_{r=1}^{\infty}$ any sequence of points of $G$. Put $e_{r}$ for the characteristic function of $a_{r} \bar{W}$. If $f$ is a measurable function on $G$ vanishing outside a compact subset of $E=\bigcup\left\{a_{r} \bar{W}: r=1,2, \cdots\right\}$ and such that

$$
\|f\|_{* p} \equiv \sum_{r=1}^{\infty}\left\|f e_{r}\right\|_{p}<\infty,
$$

then $s * f \in L^{p \prime}(G)$, and furthermore there exists a number $m_{W}^{\prime}>0$ such

$$
\|s * f\|_{p^{\prime}} \leqq m_{W}^{\prime} \cdot\|f\|_{* p} .
$$

Proof. Consider first the case in which $f$ is essentially bounded and vanishes outside $\bar{W}$. There exists then a sequence $\left(f_{n}\right)_{n=1}^{\infty}$ of functions in $C_{\bar{w}}(G)$ such that $\left\|f_{n}\right\| \leqq\|f\|_{\infty}$ and $f_{n} \rightarrow f$ a.e. By (1.1), $\left\|s * f_{n}\right\|_{1} \leqq m_{\vec{W}}\|f\|_{\infty}$ and so the $s * f_{n}$ have a weak limiting point $\mu \in M_{b}(G)$. On the other hand, since $f_{n} \rightarrow f$ in $L^{2}(G), s * f_{n} \rightarrow s * f$ in $L^{2}(G)$. It follows that $\mu=s * f \in M_{b}(G) \cap L^{2}(G) \subset L^{1}(G)$ and

$$
\|s * f\|_{1} \leqq \lim _{n \rightarrow \infty}\left\|s * f_{n}\right\|_{1} \leqq m_{\bar{W}}\|f\|_{\infty} .
$$

We also know that

$$
\|s * f\|_{2} \leqq\|s\|_{P} \cdot\|f\|_{2} \cdot
$$

Now (5.3) and (5.4) and the Riesz convexity theorem combine to show that, for some number $m_{W}^{\prime}>0$ and all $p \geqq 2$, one has

$$
\|s * f\|_{p^{\prime}} \leqq m_{W}^{\prime} \cdot\|f\|_{p}
$$

whenever $f \in L^{p}(G)$ vanishes outside $\bar{W}$. By translation, (5.5) remains valid whenever $f \in L^{p}(G)$ vanishes outside a translated set $a \bar{W}$, where $a \in G$ is arbitary.

Now suppose that $f$ vanishes outside a compact subset of $E$ and and satisfies (5.1). Then $f=\sum_{r=1}^{\infty} f_{r}$, where $f_{r}=f e_{r}$ and where the series converges in $L^{p}(G)$ and a fortiori in $L^{2}(G)$. By (5.5), 


$$
\left\|s * f_{r}\right\|_{p^{\prime}} \leqq m_{W}^{\prime} \cdot\left\|f_{r}\right\|_{p},
$$

so that in particular $\sum_{r=1}^{\infty}\left(s * f_{r}\right)$ is convergent in $L^{p^{\prime}}(G)$. This latter series is, however, convergent in $L^{2}(G)$ to $s * f$, whence it appears that $s * f \in L^{p^{\prime}}(G)$ and, from (5.6), that (5.2) is true. This completes the proof.

Remarks. (1) In the statement of (B) we assumed that $f$ vanishes outside a compact subset of $E$ merely to ensure that $s * f$ is defined a priori. Actually, the proof furnishes a method of extending the definition of $s * f$ to all cases in which $f$ vanishes outside $E$ and satisfies (5.1).

Notice that if $G=R^{n}$, we can always arrange that the $a_{r} \bar{W}$ form a covering of $R^{n}$ by nonoverlapping congruent closed $n$-dimensional cubes; this is indeed one of the most natural choices of the $a_{r} \bar{W}$ in this case. Taking $n=1$, we see that $s \in P^{1}(R)$ if and only if the operator $f \rightarrow s * f$ maps the Wiener class $M_{1}$ ([5], p. 73) into $L^{1}(R)$; and that any continuous linear operator from $M_{1}$ into $L^{1}(R)$ which commutes with translations is of the form $f \rightarrow s * f$ for a suitably chosen $s \in P^{1}(R)$.

(2) By virtue of Theorem 1, (B) expresses some nontrivial extension properties possessed by all continuous linear operators from $C_{c}(G)$ into $M_{b}(G)$ which commute with translations.

(C) In case $G=R^{n}=X$, it is simple to specify smoothness conditions on $\hat{s}$ ensuring that a given $s \in P\left(R^{n}\right)$ shall belong to $P^{1}\left(R^{n}\right)$. In fact, if we define $m_{n}$ to be 1 if $n=1$ and to be $2[n / 4]+2$ if $n>1$ (square brackets denoting the integral part), it is sufficient that each partial derivative of $\hat{s}$ of order at most $m_{n}$ be expressible as the sum of a function in $L\left(R^{n}\right)$ and a function in $L^{2}\left(R^{n}\right)$. (The partial derivatives are here understood in the distributional sense.)

\section{REFERENCES}

1. R. E. Edwards, Convolutions as bilinear and linear operators, Canad. J. Math. 16 (1964), 275-285.

2. - Bipositive and isometric isomorphisms of some convolution algebras, Canad.

J. Math. 17 (1965), 839-846.

3. G. I. Gaudry, Quasimeasures and operators commuting with convolution, To appear in Pacific J. Math.

4. W. Rudin, Fourier analysis on groups, Interscience Publishers, New York (1962).

5. N. Wiener, The Fourier integral and certain of its applications, Cambridge Univ. Press (1933).

AUSTRALIAN National University 



\section{PACIFIC JOURNAL OF MATHEMATICS}

\section{EDITORS}

\section{H. SAMELSON}

Stanford University

Stanford, California

\section{R. M. BLUMenthaL \\ University of Washington \\ Seattle, Washington 98105}

*J. DugundjI

University of Southern California Los Angeles, California 90007

\section{RICHARD ARENS}

University of California

Los Angeles, California 90024

\section{ASSOCIATE EDITORS}
E. F. BECKENBACH
B. H. NeUManN
F. WOLF
K. YosIDA

\section{SUPPORTING INSTITUTIONS}

\author{
UNIVERSITY OF BRITISH COLUMBIA \\ CALIFORNIA INSTITUTE OF TECHNOLOGY \\ UNIVERSITY OF CALIFORNIA \\ MONTANA STATE UNIVERSITY \\ UNIVERSITY OF NEVADA \\ NEW MEXICO STATE UNIVERSITY \\ OREGON STATE UNIVERSITY \\ UNIVERSITY OF OREGON \\ OSAKA UNIVERSITY \\ UNIVERSITY OF SOUTHERN CALIFORNIA
}

\author{
STANFORD UNIVERSITY \\ UNIVERSITY OF TOKYO \\ UNIVERSITY OF UTAH \\ WASHINGTON STATE UNIVERSITY \\ UNIVERSITY OF WASHINGTON \\ AMERICAN MATHEMATICAL SOCIETY \\ CHEVRON RESEARCH CORPORATION \\ TRW SYSTEMS \\ NAVAL ORDNANCE TEST STATION
}

Mathematical papers intended for publication in the Pacific Journal of Mathematics should be typewritten (double spaced). The first paragraph or two must be capable of being used separately as a synopsis of the entire paper. It should not contain references to the bibliography. Manuscripts may be sent to any one of the four editors. All other communications to the editors should be addressed to the managing editor, Richard Arens at the University of California, Los Angeles, California 90024.

50 reprints per author of each article are furnished free of charge; additional copies may be obtained at cost in multiples of 50 .

The Pacific Journal of Mathematics is published monthly. Effective with Volume 16 the price per volume (3 numbers) is $\$ 8.00$; single issues, $\$ 3.00$. Special price for current issues to individual faculty members of supporting institutions and to individual members of the American Mathematical Society: $\$ 4.00$ per volume; single issues $\$ 1.50$. Back numbers are available.

Subscriptions, orders for back numbers, and changes of address should be sent to Pacific Journal of Mathematics, 103 Highland Boulevard, Berkeley 8, California.

Printed at Kokusai Bunken Insatsusha (International Academic Printing Co., Ltd.), No. 6, 2-chome, Fujimi-cho, Chiyoda-ku, Tokyo, Japan.

PUBLISHED BY PACIFIC JOURNAL OF MATHEMATICS, A NON-PROFIT CORPORATION

The Supporting Institutions listed above contribute to the cost of publication of this Journal, but they are not owners or publishers and have no responsibility for its content or policies.

* Paul A. White, Acting Editor until J. Dugundji returns. 


\section{Pacific Journal of Mathematics}

\section{Vol. 16, No. 2 December, 1966}

Loren N. Argabright, Invariant means on topological semigroups ........ 193

William Arveson, A theorem on the action of abelian unitary groups ...... 205

John Spurgeon Bradley, Adjoint quasi-differential operators of Euler

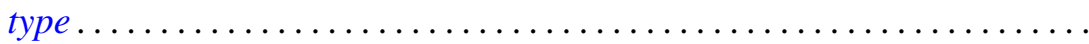

Don Deckard and Lincoln Kearney Durst, Unique factorization in power series rings and semigroups ........................... 239

Allen Devinatz, The deficiency index of ordinary self-adjoint differential operators..................................... 243

Robert E. Edwards, Operators commuting with translations ............ 259

Avner Friedman, Differentiability of solutions of ordinary differential equations in Hilbert space .............................. 267

Boris Garfinkel and Gregory Thomas McAllister, Jr., Singularities in a variational problem with an inequality ......................

Seymour Ginsburg and Edwin Spanier, Semigroups, Presburger formulas,

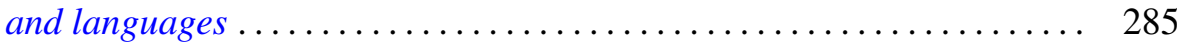

Burrell Washington Helton, Integral equations and product integrals . . . . . . 297

Edgar J. Howard, First and second category Abelian groups with the n-adic topology.........................................

Arthur H. Kruse and Paul William Liebnitz, Jr., An application of a family homotopy extension theorem to ANR spaces.

Albert Marden, I. Richards and Burton Rodin, On the regions bounded by homotopic curves

Willard Miller, Jr., A branching law for the symplectic groups ...

Marc Aristide Rieffel, A characterization of the group algebras of the finite

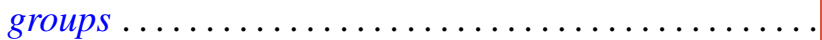

P. P. Saworotnow, On two-sided $H^{*}$-algebras

John Griggs Thompson, Factorizations of p-solvable groups ...

Shih-hsiung Tung, Harnack's inequalities on the classical Cartan

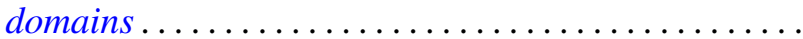

